\title{
Effects Of Variable Viscousity On Boundary Layer Flow With Convective Surface Boundary Condition
}

\author{
Disu Akeem B.,Olorunnishola Toyin \& Ishola Christie Y. \\ School of Science and Technology, National Open University of Nigeria \\ Victoria Island, Lagos, Nigeria
}

\begin{abstract}
This study was conducted to investigate the effects of variable viscousity on boundary layer flow with convective surface boundary condition. The governing equations were transformed into a system of ordinary differential equations by using similarity variable. The system of ordinary differential equations were solved numerically by using the sixth-order Runge-kutta integration scheme with a modified version of the Newton-Raphson shooting method.The results were presented graphically, skin friction and Nusselt number were tabulated.
\end{abstract}

Keywords: Boundary layer flow, Convective boundary condition, Flat Plate and Variable viscousity

\section{Introduction}

The study of convective heat transfer in a viscous imcompressible fluid over flat plate has been received considerable attention due to it application in processes involving high temperature such as gas turbines, nuclear, power plant and thermal storage.The problem of a fluid flow over a horizontal, stationary flat plate in uniform free stream was first solved by Blausis [1]. This was done by transforming the governing partial differential equations into ordinary differential equations by introducing a new independent variable called the similarity variable. The similarity variable has been applied to solve thermal boundary layer for the constant surface temperature at the plate [2].

Aziz [3] solved laminar thermal boundary layer flow over a flat plate with convective surface boundary condition by applied similarity variable and presented the Biot number on the heat transfer characteristics. The steady laminar boundary layer flow of a non-Newtonian fluid over an impermeable flat plate with convective boundry condition was investigated by Gabriella and Krisztian[4], the power law index of the fluid was considered.

Since the effects of some fluid properties such as viscousity varies with temperature in most fluid, the study of laminar thermal boundary layer flow over a flat plate with convective surface boundary condition need a more robust model for viscousity, taking into account variability of viscousity with temperature. In fact, all the above studies assumed that viscousity does not vary with temperature which is not true in practical situation. As a result of this, we present the effects of variable viscousity on laminar boundary layer flow over a flat with convective surface boundary condition.

\section{Mathematical Formulation}

We consider two-dimensional steady incompressible fluid flow with heat transfer by convection over a flat plate. A stream of cold fluid at temperature moving over the right surface of the plate with a uniform velocity while the left surface of the plate is heated by convection from a hot fluid at temperature. We assumed that the fluid viscousity varies as exponential function of temperature $\mathrm{T}$, in the form

$$
\mu(T)=\mu e^{-\gamma \theta}
$$

(1)

Salem \& Odda [5]

where $\gamma$ is variable viscosity parameter

$$
\begin{gathered}
\frac{\partial u}{\partial x}+\frac{\partial v}{\partial y}=0 \\
\rho\left(u \frac{\partial u}{\partial x}+v \frac{\partial u}{\partial y}\right)=\frac{\partial}{\partial y}\left(\mu(T) \frac{\partial u}{\partial y}\right)
\end{gathered}
$$


$\rho C p\left(u \frac{\partial T}{\partial x}+v \frac{\partial T}{\partial y}\right)=k \frac{\partial^{2} T}{\partial y^{2}}$

The boundary conditions relevant to the problem are taken as follows:

$u(x, 0)=v(x, 0)=0$

$u(x, \infty)=T_{\infty}$

We assumed that the bottom surface is heated by convection from a hot fluid at temperature $T_{f}$ which provides a heat transfer coefficient $h_{f}$. The boundary conditions at the plate surface and far into the cold fluid may be written as

$-k \frac{\partial T}{\partial y}(x, 0)=h_{f}\left[T_{f}-T(x, 0)\right]$

$T(x, \infty)=T_{\infty}$

where $u, v$ are the velocity components along $x, y$ coordinates, $\mathrm{p}$ the density of the fluid, $\mu$ the kinematic viscousity, $k$ the thermal conductivity, $C p$ the specific heat at constant pressure and $T$ the fluid temperature.

A similarity solution of Equations (1)-(6) is obtained by defining an independent variable $\eta$ and a dependent variable in terms of stream function $\psi(x, y)$ such that

$u=\frac{\partial \psi}{\partial y}, v=-\frac{\partial \psi}{\partial x}$

$\eta=y \sqrt{\frac{U_{\infty}}{2 v x}}, \psi=\sqrt{2 v x U_{\infty}} f(\eta), T=\left(T_{f}-T_{\infty}\right) \theta(\eta)+T_{\infty}$

With the similarity solution, we obtained ordinary differential equations for the functions $f(\eta)$ and $\theta(\eta)$ as

$f^{\prime \prime \prime}+e^{\gamma \theta} f f^{\prime \prime}-\not f^{\prime \prime} \theta^{\prime}=0$

$\theta^{\prime \prime}+\operatorname{Pr} f \theta^{\prime}=0$

and the boundary conditions become

$f(0)=0, f^{\prime}(0)=0, \theta^{\prime}(0)=-B i[1-\theta(0)]$
$f^{\prime}(\infty)=1, \theta(\infty)=0$

Where $\operatorname{Pr}=\frac{\rho C p}{k}, B i=\frac{h f}{k} \sqrt{\frac{v x}{U_{\infty}}}$

$\mathrm{Pr}$ is the prandtl number and $B i$ is the Biot number.

\section{Skin Friction And Nusselt Number}

The shear stress along the wall (wall skin friction) is defined as $\tau=\left.\mu \frac{\partial u}{\partial y}\right|_{y=0}$,then the skin friction can be defined

as

$\tau=f^{\prime \prime}(0)$

The rate of heat transfer at the wall is defined by $N u=-\left.k \frac{\partial T}{\partial y}\right|_{y=0}$, therefore, Nusselt Number can be written as $N u=-\theta^{\prime}(0)$

\section{Discussion Of Results}

Equations (9) and (10) with boundary conditions (12) were solved numerically by using the sixthorder Runge-kutta integration scheme with a modified version of the Newton-Raphson shooting with $\gamma, \operatorname{Pr}$ and 
$B i$ as prescribed parameters. The series of computation were performed using Maple [6] which is a symbolic and computation language.

As an accuracy mathematical check, setting $\gamma=0$, the work of Aziz [3] is fully recovered. Numerical computations were performed and the results were presented graphically in Figure 1-3 shown the interesting features of significant parameters on velocity and temperature distributions. Throughout the computations, we employed default values $\gamma=0.1, \operatorname{Pr}=0.72, \mathrm{Bi}=0.1$ unless otherwise stated.

Figure 1 depicts the velocity profile with variation of variable viscousity parameter $(\gamma)$. It is observed that an increase in variable viscousity causes a fall in the velocity profile.

Figures 2 and 3 show the temperature profile for different values of Biot number (Bi) and Prandtl number (Pr). It can be seen that an increase in Bi produces a rise in the temperature profile. But, an increase in Pr causes a fall in the temperature profile.

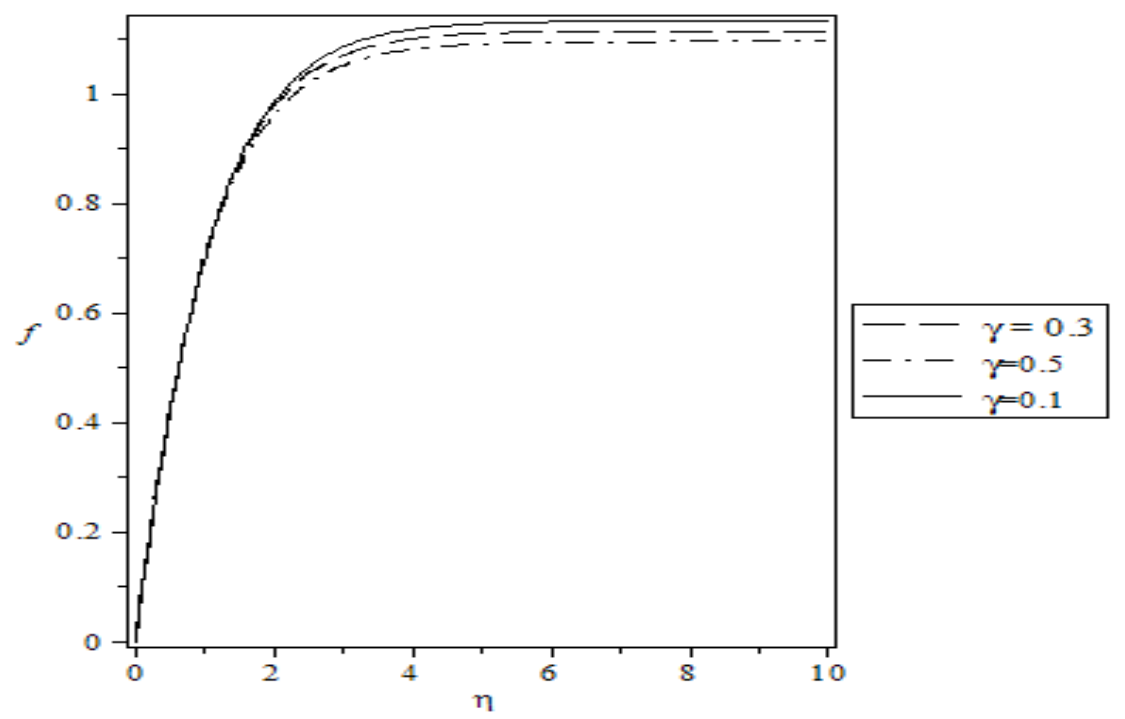

Figure 1: Velocity profile for different values $\gamma$

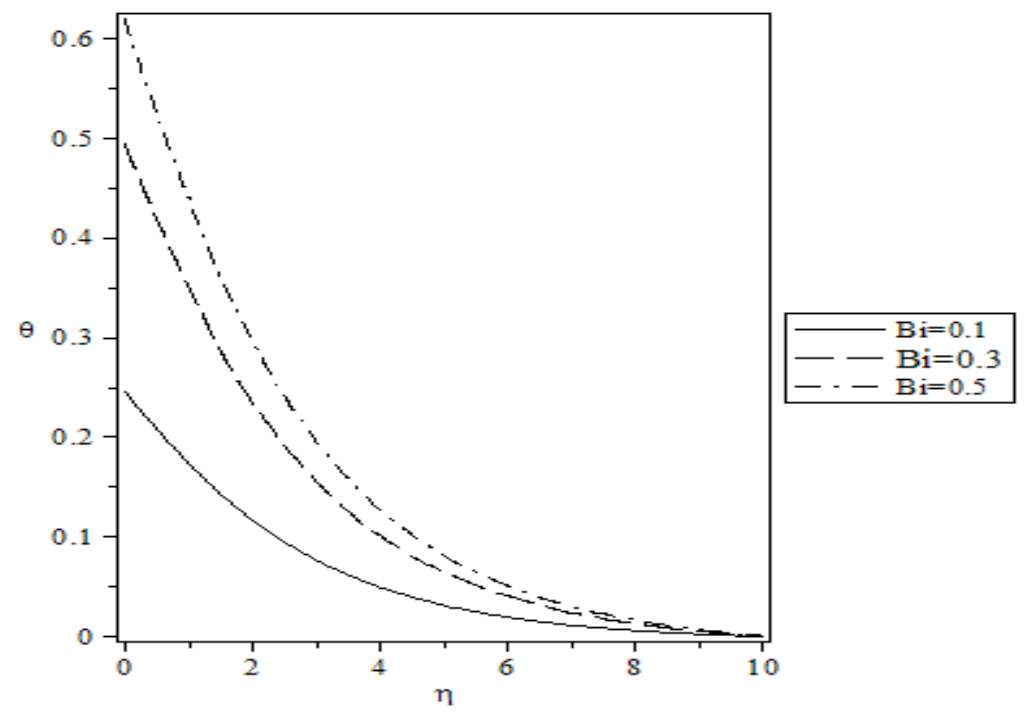

Figure 2: Temperature profile for different values Bi 


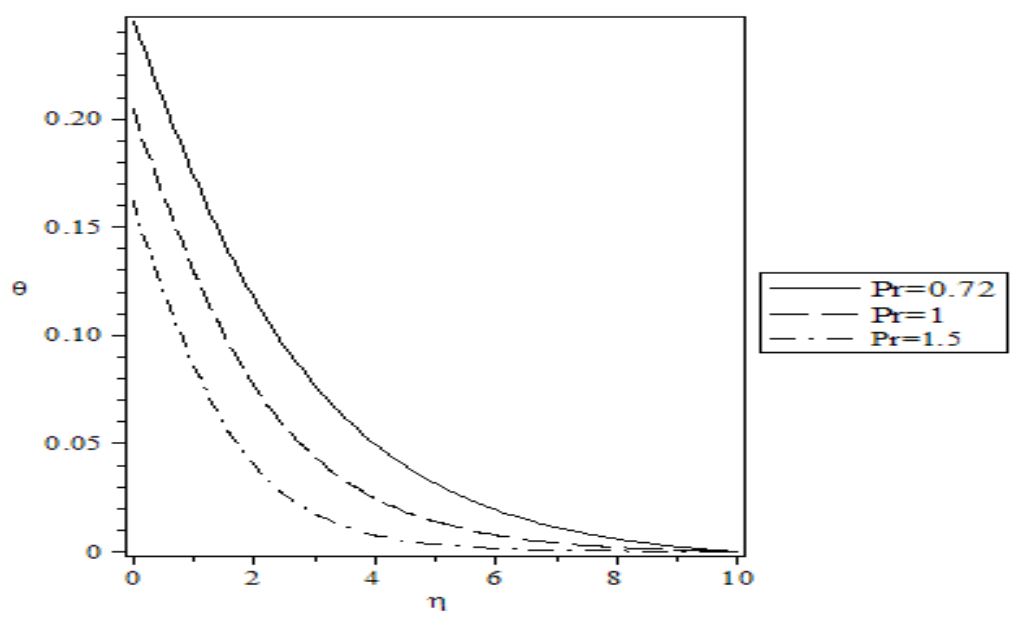

Figure 3: Temperature profile for different values of $\operatorname{Pr}$

Table 1 represents values of the skin friction coefficient $f^{\prime \prime}(0)$ and heat transfer rates $-\theta^{\prime}(0)$ for different values of variable viscosity parameter $\gamma$, Biot number $B i$ and Prandtl number $\operatorname{Pr}$. It can be seen clearly that an increase in $\gamma$ decreases $f^{\prime \prime}(0)$ and $-\theta^{\prime}(0)$. It can be observed that an increase in $B i$ increases $-\theta^{\prime}(0)$, but causes a decrease in $f^{\prime \prime}(0)$. Increase in $\operatorname{Pr}$ causes a decrease in $f^{\prime \prime}(0)$ but produces an increase in $-\theta^{\prime}(0)$.

\begin{tabular}{|l|l|l|l|}
\hline \multirow{4}{*}{$\gamma$} & & $f^{\prime \prime}(0)$ & $-\theta^{\prime}(0)$ \\
\hline \multirow{3}{*}{$B i$} & 0.1 & -0.636420 & 0.0754928 \\
\cline { 2 - 4 } & 0.3 & -0.654611 & 0.0753200 \\
\cline { 2 - 4 } & 0.5 & -0.673468 & 0.0751405 \\
\cline { 2 - 4 } & 0.1 & -0.636420 & 0.0754928 \\
\cline { 2 - 4 } & 0.3 & -0.645534 & 0.1516346 \\
\hline \multirow{3}{*}{$\operatorname{Pr}$} & 0.5 & -0.650170 & 0.1897817 \\
\cline { 2 - 4 } & 0.72 & -0.636420 & 0.0754928 \\
\cline { 2 - 4 } & 1 & -0.635169 & 0.0795528 \\
\cline { 2 - 4 } & 1.5 & -0.633851 & 0.0837897 \\
\hline
\end{tabular}

\section{Conclusion}

This paper studied effects of variable viscousity on boundary layer flow with convective surface boundary condition. The governning equations were transformed into a system of ordinary differential equations using similarity variable. The results were presented graphically and effects of the variable viscosity parameter, Biot number and Prandtl number were discussed. The skin friction, Nusselt number at the plate surface were also discussed. Hence, there is a good agreement between the present study and a similarity solution for laminar thermal boundary layer over a flat plate with a convective surface boundary condition by Aziz [3] when $\gamma=0$

\section{References}

[1]. H. Blasius, Grenzschichten in flussigkeiten mit kleiner reibung, Z. Math Phys., 56, 1908, 1-37.

[2]. F.P. Incropera et al, Fundamental of heat and mass transfer (New York: John Wiley, 2007). (6)

[3]. A. Aziz, A similarity Solution for laminar thermal boundary layer over a flat plate with a convective surface boundary condition, Commun. Nonlinear Sci. Numer. Simul., 14, 2009, 1064-1068.

[4]. B. Gabriella and H. Krisztian Acta Polytechnica Hungarica 8(6), 2011

[5]. A. M. Salem and S. N. Odda, Influence of thermal conductivity and variable viscosity on the flow of micro-polar fluid past a continuously moving plate with suction or Injection. The Korean Society for Industrial and Applied Mathematics, (2005).

[6]. A. Heck, Introduction to Maple (Springer-Verlag, New York, 2003). (3) 